\title{
Microvascular decompression of the trigeminal nerve in the treatment of SUNCT and SUNA
}

\author{
Max Williams, ${ }^{1}$ Renata Bazina, ${ }^{2}$ Leong Tan, ${ }^{2}$ Hal Rice, ${ }^{3}$ Simon A Broadley ${ }^{1,4}$
}

${ }^{1}$ Department of Neurology, Gold Coast Hospital, Southport,

Australia

2Department of Neurosurgery, Gold Coast Hospital, Southport, Australia

${ }^{3}$ Department of Medical Imaging, Gold Coast Hospital, Southport, Australia

${ }^{4}$ School of Medicine, Gold Coast Campus, Griffith University, Australia

\section{Correspondence to}

Dr Max Williams, Department of Neurology, Gold Coast Hospital, 108 Nerang Street, Southport 0 4215, Australia;

maxhw@austarnet.com.au

Received 6 May 2009

Revised 8 December 2009

Accepted 2 January 2010

\section{ABSTRACT}

Background Medical management of short-lasting unilateral neuralgiform headache with conjunctival injection and tearing (SUNCT) syndrome and short-lasting unilateral neuralgiform headache attacks with cranial autonomic symptoms (SUNA) is often unsatisfactory.

Methods The authors report nine cases of SUNCT/ SUNA that failed medical treatment and had an aberrant arterial loop either in contact with or compressing the appropriate trigeminal nerve demonstrated on MRI. All underwent microvascular decompression of the ipsilateral trigeminal nerve for intractable pain.

Results Immediate and complete relief of SUNCT and SUNA symptoms occurred in 6/9 (67\%) cases. This was sustained for a follow-up period of 9-32 months (mean 22.2). In 3/9 (33\%) cases, there was no benefit. Ipsilateral hearing loss was observed in one case. Conclusion Medically intractable SUNCT and SUNA subjects with a demonstrable aberrant arterial loop impinging on the trigeminal nerve on neuroimaging may benefit from microvascular decompression.

\section{INTRODUCTION}

Short-lasting unilateral neuralgiform headache with conjunctival injection and tearing (SUNCT) and short-lasting unilateral neuralgiform headache attacks with cranial autonomic symptoms (SUNA) are primary headache disorders which are often debilitating. The pain is typically in the distribution of the first division of the trigeminal nerve. The International Headache Society (IHS) classification has defined SUNCT syndrome as having brief headache attacks lasting 5-240 s with an attack frequency of 3-200 per day with associated ipsilateral prominent autonomic features, ${ }^{1}$ while SUNA allows a broader range of attack duration, from $2 \mathrm{~s}$ to $10 \mathrm{~min}$, and autonomic features may be relatively minor. ${ }^{1}$ Originally thought of as very rare conditions, recent estimates of incidence and prevalence are not insignificant. ${ }^{2}$

The IHS recognises episodic and chronic forms of SUNA, but as yet no such distinction has been made for SUNCT. Episodic SUNA refers to attacks occurring in periods lasting 7 days to 1 year separated by pain-free intervals lasting 1 month or longer, whereas chronic indicates attacks occurring for more than 1 year without remissions or with remission periods of less than 1 month. ${ }^{1}$ The value of categorising SUNCT along similar lines as either episodic or chronic in determining prognosis and therapeutic approach has been highlighted. ${ }^{2}$

Lamotrigine is currently the treatment of choice for SUNCT and SUNA ${ }^{3}$ but appears to be only partially effective in those with a chronic pattern. ${ }^{2}$ Lignocaine infusion can also be effective but is in general only a short-term solution. ${ }^{24}$ Despite these therapies, many patients prove to have intractable attacks of pain.

One of the principal differential diagnoses of SUNCT/SUNA is trigeminal neuralgia. First division involvement in trigeminal neuralgia is uncommon, being seen in approximately $9 \%$ of cases, ${ }^{5}$ but can occasionally be associated with mild autonomic features, such as tearing. ${ }^{6}$ SUNCT and SUNA are differentiated from first division trigeminal neuralgia by the prominence of the autonomic features (tearing, eye lid oedema, rhinorrhoea and conjunctival injection), longer duration of attacks, the absence of a refractory period to trigger factors and relative lack of response to carbamazepine. ${ }^{17-9}$

Microvascular decompression of the trigeminal nerve, or the Jannetta procedure, is an accepted mode of treatment for trigeminal neuralgia. ${ }^{10-12}$ This approach was based on the opinion of one of the first proponents of the vascular theory for trigeminal neuralgia, Walter Dandy in $1934 .{ }^{13}$ Whether or not an aberrant vessel is compressing the trigeminal nerve, trigeminal neuralgia is classified by the IHS as "classical." The term 'symptomatic' is reserved for trigeminal neuralgia with causes other than vascular compression by an aberrant vascular loop. ${ }^{14}$

An aberrant vascular loop in contact with the trigeminal nerve is demonstrable in $47-90 \%$ of cases of trigeminal neuralgia ${ }^{15}{ }^{16}$ with the frequency increasing with greater MRI resolution. ${ }^{5}$ While anecdotal cases are reported of similar aberrant vascular loops in SUNCT, the actual frequency of this is uncertain. In a series of 52 cases of SUNCT and SUNA, only three $(7 \%)$ had vascular compression, but it was not stated whether high-resolution views of the trigeminal nerve were performed. ${ }^{17}$ It has been suggested that the finding of an aberrant vascular loop should be regarded as an incidental finding. ${ }^{17}$ Recently, we reported that of 17 cases with SUNCT or SUNA imaged at $1.5 \mathrm{~T}$ with highresolution T2 constructive interference in the steady-state (CISS) images of the posterior fossa, 15 (88\%) cases had an aberrant arterial loop in contact with the clinically relevant trigeminal nerve. ${ }^{2}$

Neither the IHS classification ${ }^{1}$ nor the proposed classification by Cohen et al ${ }^{17}$ of SUNCT/SUNA mentions whether an aberrant vascular loop compressing the trigeminal nerve should be regarded as a symptomatic or secondary cause.

A number of case reports have described successful microvascular decompression of the trigeminal nerve in SUNCT. ${ }^{15}$ 18-20 However, caution has been advised with this approach due to complications. ${ }^{21}$ 
Here, we report outcomes in nine cases of SUNCT/SUNA in whom microvascular decompression, using a modified Jannetta procedure, was performed as a last resort for intractable pain.

\section{METHODS \\ Subjects}

Cases met the IHS criteria for SUNCT or SUNA, ${ }^{1}$ and all had prominent autonomic features. All subjects had been referred to neurology and neurosurgical clinics in the Gold Coast region of Australia. Subjects with intractable pain resistant to standard medical therapy were offered the option of surgery.

\section{Imaging}

MRI scans were performed on Siemens Symphony (Siemens, Erlangen, Germany) or GE Signa (GE Healthcare Global Diagnostic Imaging, Waukesha, Wisconsin, USA) $1.5 \mathrm{~T}$ machines with high-resolution T2 CISS sequences of the posterior fossa. Only cases with a demonstrable vascular loop either in contact with or distorting the nerve on the clinically relevant side were offered surgery (figure 1).

\section{Surgical technique}

A modified Jannetta procedure ${ }^{22}$ was used as follows: under anaesthesia, the subject was placed in the lateral position with the neck flexed. The head was rotated slightly away from the affected side and placed in Mayfield pins three-point fixation. Stereotactic navigation was used to identify transverse and sigmoid sinus landmarks. A retro sigmoid approach was utilised with a $5 \mathrm{~cm}$ skin incision behind the mastoid and a $32 \mathrm{~mm}$ diameter size craniotomy, the apex of which was at the venous angle. The dura was opened in a T fashion and hitched. CSF was aspirated to facilitate cerebellar retraction. An operating microscope was brought in, and a Yasargil retractor was applied to the superolateral surface of the cerebellum (figure 2A). The arachnoid adhesions were dissected to the petrous ridge and the petrosal vein ligated allowing the trigeminal dorsal root entry zone to be identified and freed from any adhesions (figure $2 \mathrm{~B}$ ). The vessel loop was dissected from the nerve, and a silicone cuff cut to size from a $6 \mathrm{~mm}$ diameter scleral buckling component with the centre cored out and an open side cut was placed around the trigeminal nerve root (figure $2 \mathrm{C}$ ).
Haemostasis was secured, the dura closed and the bone flap returned. The subcutaneous tissue and skin were closed in standard fashion. Postop MRI showed the silicone cuff around the trigeminal root entry zone and decompression from the vascular loop (figure 2D).

\section{RESULTS}

\section{Baseline characteristics}

Of 24 subjects with SUNCT/SUNA identified over a 6-year period, nine had intractable pain that was resistant to medical therapy including lamotrigine at doses of 300-600 mg daily and lignocaine infusion. These subjects were offered surgery, and all agreed to proceed with this treatment option.

The baseline characteristics and preoperative treatment of these patients are summarised in table 1 . The mean age of subjects was 55 years (range 48-71 years). The sex distribution was fairly even (five males, four females). All but two cases had chronic SUNCT/ SUNA. In five cases, there were just SUNCT attacks, in two, there were only SUNA attacks, and in two, there was a mixture of SUNCT and SUNA. The mean duration of symptoms prior to surgery was 5.7 years (range 1 month-26 years). The split between attack patterns was evenly distributed, with three each having saw-toothed, stab groups and single stab patterns.

All cases had been trialled on a variety of analgesic and anticonvulsant medications (table 1). One subject with SUNA had been symptomatic for only 1 month but became suicidal with the pain. One male subject had SUNCT and responded well to lamotrigine at $100 \mathrm{mg}$ per day but developed a severe allergic skin reaction. Other medications, including topiramate, failed to control his attacks. Another male subject with SUNA opted for surgery because of severe pain despite high doses of carbamazepine, phenytoin and narcotics. This subject also became suicidal and did not wish to try other medication. A subcutaneous lignocaine infusion ${ }^{2}$ was used in seven cases to control pain prior to surgery with an incomplete or only short-lived effect.

\section{Outcomes from surgery}

At surgery an aberrant arterial loop was found in all nine cases. This was the superior cerebellar artery in seven and the anterior
Figure 1 High resolution T2 constructive interference in the steadystate images of a patient with left sided short-lasting unilateral neuralgiform headache with conjunctival injection and tearing showing an aberrant loop of the posterior inferior cerebellar artery abutting the left $\mathrm{V}$ nerve (arrows).
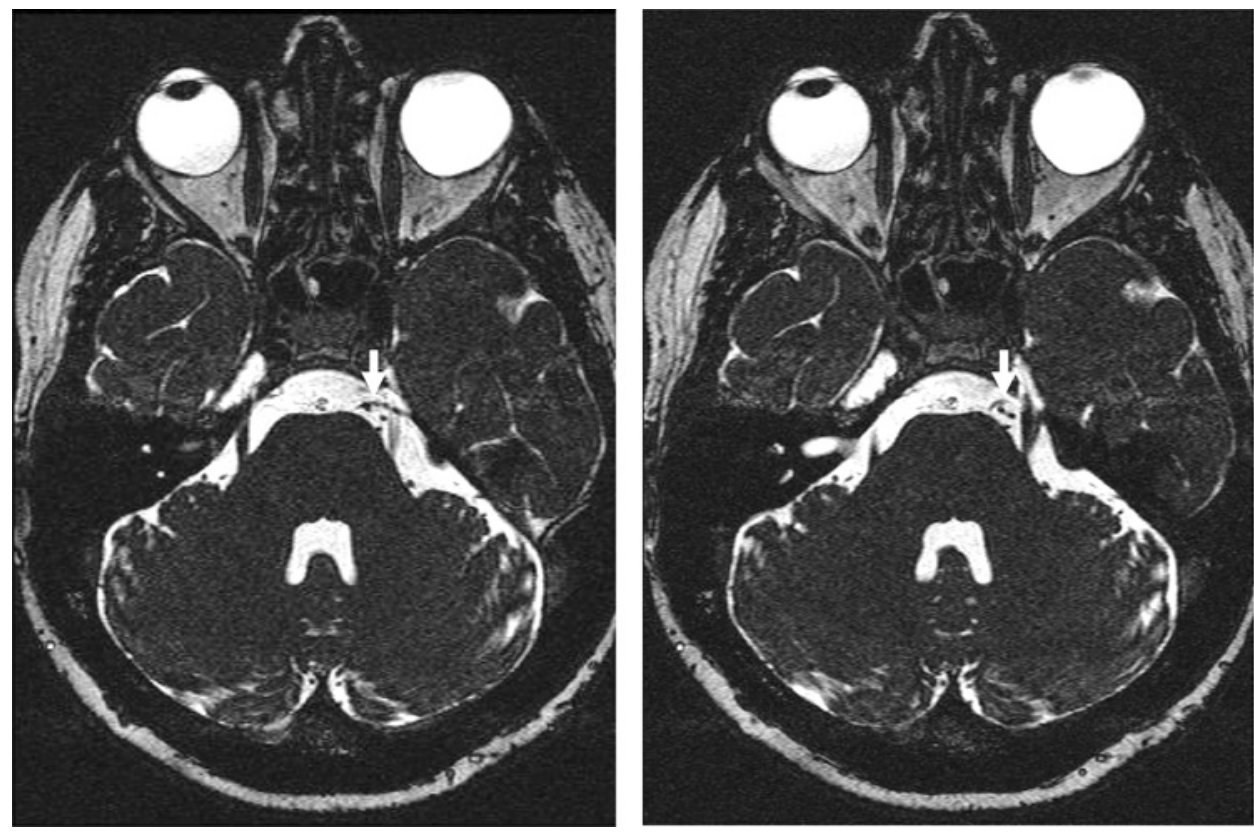
Figure 2 Intraoperative microphotographs showing $(A)$ exposure of the $V$ nerve, $(B)$ dissection of the aberrant artery (arrow) from the nerve, $(C)$ placement of the silicone cuff and (D) MRI brain showing the silicone cuff (arrow) in situ postprocedure.
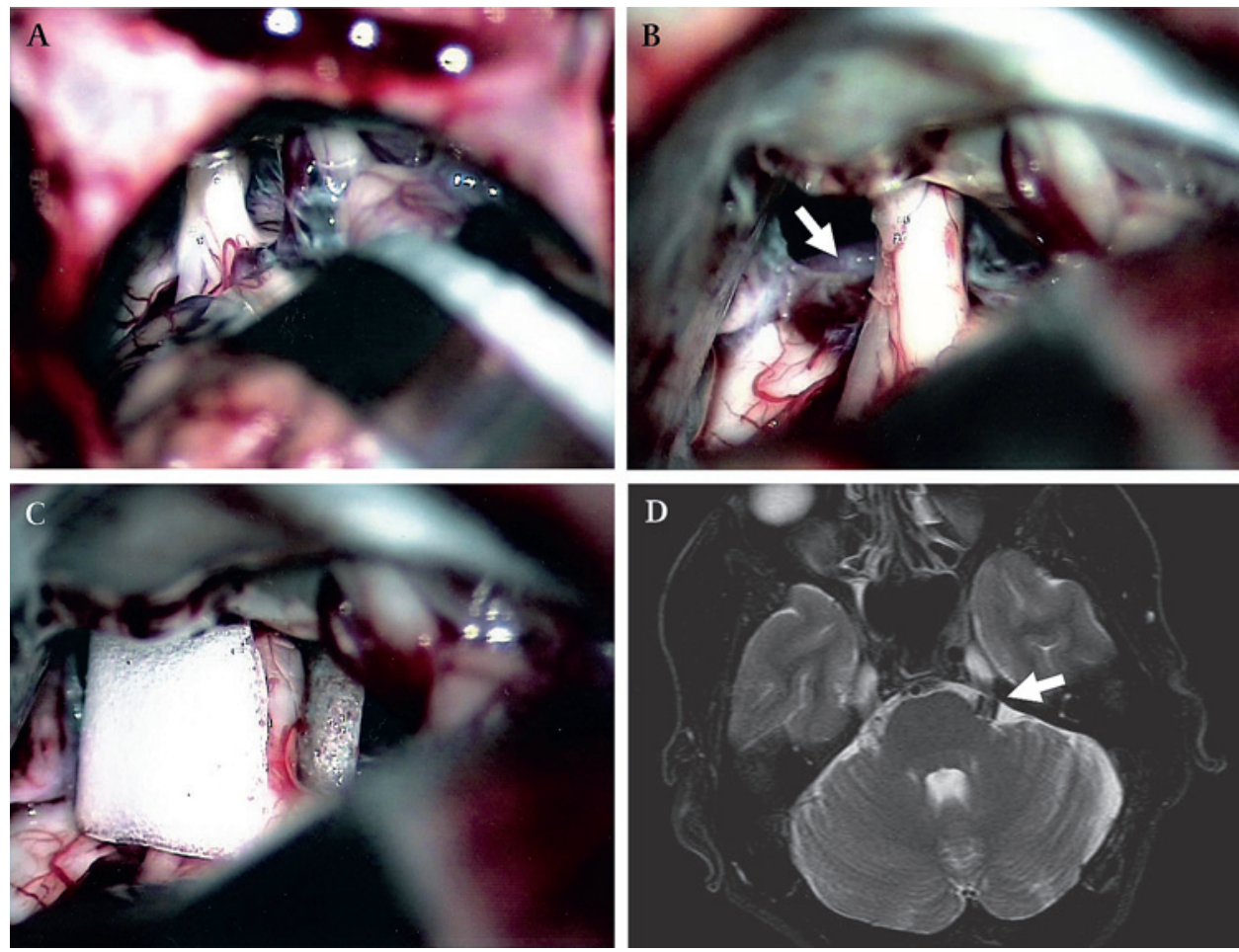

inferior cerebellar artery in two. A vein was also seen in contact with the trigeminal nerve in two cases and arachnoid adhesions in one. The findings at surgery and the subsequent outcomes are summarised in table 2 .

In $6 / 9(67 \%)$ cases, there was complete relief of SUNCT/ SUNA symptoms immediately following surgery, and this has been sustained for a follow-up period of 9-32 months (mean 22.2 months). Three of these cases continue on medication, one on lamotrigine and topiramate for epilepsy and migraine, one on topiramate alone for epilepsy and migraine, and the other on lamotrigine for epilepsy and central pain disorder. Two cases continue to have autonomic features without pain triggered by previously noted trigger factors. In both cases, these were not troublesome, consisting only of mild ipsilateral tearing or rhinorrhoea.
In one-third of cases, the outcome was unsatisfactory. SUNA attacks in one case continue with no significant change. One case (case 8) with alternating bilateral attacks was operated on the more symptomatic side and noted relief of her chronic SUNA for 2 weeks, but attacks recurred with the same frequency thereafter. Another case who had ophthalmic herpes zoster just prior to her ipsilateral chronic SUNA attacks had resolution of the autonomic features but intermittent pain over the first division of the trigeminal nerve persists as before.

\section{Complications}

Postoperative wound infection that completely resolved with antibiotics was seen in one case. Two cases had diminished sensation of the ipsilateral first and second divisions of the

Table 1 Patient characteristics and preoperative management

\begin{tabular}{|c|c|c|c|c|c|c|c|c|c|c|c|}
\hline Case & $\begin{array}{l}\text { Age } \\
\text { (years) }\end{array}$ & Sex & Type & $\begin{array}{l}\text { Symptom } \\
\text { duration (years)* }\end{array}$ & $\begin{array}{l}\text { Attack frequency } \\
\text { (per day) }\end{array}$ & $\begin{array}{l}\text { Attack } \\
\text { duration (s) }\end{array}$ & $\begin{array}{l}\text { Trigger } \\
\text { factors }\end{array}$ & $\begin{array}{l}\text { Attack } \\
\text { pattern }\end{array}$ & Prior medication & $\begin{array}{l}\text { Lignocaine } \\
\text { response } †\end{array}$ & $\begin{array}{l}\text { MRI } \\
\text { findings }\end{array}$ \\
\hline 1 & 71 & $\mathrm{M}$ & SUNCT & 6 & $30-200$ & $60-120$ & Yes & $\begin{array}{l}\text { Saw- } \\
\text { toothed }\end{array}$ & $\begin{array}{l}\text { CBZ/LTG/GBP/BCF/ } \\
\text { PRED }\end{array}$ & $+++(\times 3)$ & SCA \\
\hline 2 & 54 & M & $\begin{array}{l}\text { SUNCT/ } \\
\text { SUNA }\end{array}$ & 0.1 & $15-20$ & $1-300$ & Yes & Stab groups & CBZ/PHT/GBP/BCF & $\mathrm{N} / \mathrm{A}$ & SCA \\
\hline 3 & 46 & M & SUNCT & 3 & $20-30$ & $30-120$ & No & Stab groups & VPA/LTG/TPM & +++ & $\begin{array}{l}\text { AICA } \\
\text { Vein } \\
\text { Adhesions }\end{array}$ \\
\hline 4 & 61 & M & $\begin{array}{l}\text { SUNCT/ } \\
\text { SUNA }\end{array}$ & 3 & $5-10$ & $60-300$ & No & Single Stabs & PHT/LTG & ++ & $\begin{array}{l}\text { SCA } \\
\text { Vein }\end{array}$ \\
\hline 5 & 56 & $\mathrm{M}$ & SUNA & 1 & $20-30$ & $120-300$ & Yes & Single Stabs & CBZ/LTG & +++ & SCA \\
\hline 6 & 48 & $\mathrm{~F}$ & SUNA & 2 & $30-50$ & 300 & Yes & Stab groups & GBP/LTG & +++ & $\begin{array}{l}\text { AICA } \\
\text { Vein }\end{array}$ \\
\hline 7 & 51 & $\mathrm{~F}$ & SUNCT & 5 & $90-120$ & $3-10$ & Yes & $\begin{array}{l}\text { Saw- } \\
\text { toothed }\end{array}$ & $\mathrm{O}_{2} / \mathrm{PRED} / \mathrm{MPH} / \mathrm{LTG}$ & $+++(\times 2)$ & SCA \\
\hline 8 & 49 & $\mathrm{~F}$ & SUNCT & 5 & $100-300$ & $30-120$ & Yes & $\begin{array}{l}\text { Saw- } \\
\text { toothed }\end{array}$ & $\begin{array}{l}\mathrm{O}_{2} / \mathrm{IDM} / \mathrm{PETH} / \mathrm{LTG} / \\
\text { TPM }\end{array}$ & $+++(\times 3)$ & SCA \\
\hline 9 & 49 & $\mathrm{M}$ & SUNCT & 26 & $81-10$ & $20-180$ & Yes & Single Stabs & $\mathrm{O}_{2} / \mathrm{LTG} / \mathrm{TPM}$ & N/A & SCA \\
\hline
\end{tabular}

*Duration of symptoms from onset to time of surgery.

++++ , complete control of symptoms;,$++>50 \%$ control of symptoms.

AICA, anterior inferior cerebellar artery; BCF, baclofen; CBZ, carbamazepine; GBP, gabapentin; IDM, indomethacin; LTG, lamotrigine; MPH, morphine; PETH, pethidine; PHT, phenytoin; PRED, prednisolone; SCA, superior cerebellar artery; SUNA, short-lasting unilateral neuralgiform headache with autonomic symptoms; SUNCT, short-lasting unilateral neuralgiform headache with conjunctival injection and tearing; TPM, topiramate; VPA, valproic acid. 
Table 2 Outcome from surgery

\begin{tabular}{|c|c|c|c|c|c|c|}
\hline Case & Findings at surgery & Complications & Postop response & Follow-up status & $\begin{array}{l}\text { Duration of } \\
\text { follow-up (months) }\end{array}$ & Patient satisfaction* \\
\hline 1 & SCA & None & No attacks & SUNCT-free & 32 & +++ \\
\hline 2 & SCA & Wound infection & No attacks & SUNCT/SUNA free & 32 & +++ \\
\hline 4 & $\begin{array}{l}\text { SCA } \\
\text { Vein }\end{array}$ & Transient vertigo & SUNA attacks & SUNA persists & 22 & - \\
\hline 7 & SCA & None & No attacks & SUNCT-free & 10 & +++ \\
\hline 8 & SCA & None & No attacks for several weeks & SUNCT persists & 10 & - \\
\hline 9 & SCA & None & No attacks & SUNCT-free & 9 & +++ \\
\hline
\end{tabular}

${ }^{*}+++$, extremely satisfied; ++ , very satisfied; + , satisfied;,- not satisfied.

AICA, anterior inferior cerebellar artery; SCA, superior cerebellar artery; SUNA, short-lasting unilateral neuralgiform headache with autonomic symptoms; SUNCT, short-lasting unilateral neuralgiform headache with conjunctival injection and tearing.

trigeminal nerve, but this was present prior to surgery. Three cases, with pre-existing migraine, had pounding ipsilateral hemicrania with photophobia and phonophobia with nausea, vomiting and vertigo lasting $24-48 \mathrm{~h}$ in the immediate postoperative period. Ipsilateral hearing loss occurred as a consequence of the surgery in $1 / 9(11 \%)$ cases. Transient trigeminal motor weakness was seen in one case after surgery.

\section{Illustrative case}

A 71-year-old Caucasian female was admitted to hospital, complaining of right orbital and infraorbital pain in attacks lasting 1-3 min occurring 30-200 times a day in a saw-toothed pattern over the previous 6 years. Attacks were associated with conjunctival injection, tearing, ptosis, eyelid oedema and nasal congestion. Trigger factors included light touch to the face, chewing, talking and teeth cleaning. There was no refractory period to trigger stimuli, and weight loss was noted. Treatment with carbamazepine at the highest tolerated dose of $800 \mathrm{mg}$ per day had been ineffective. Twelve months prior to admission, a diagnosis of SUNCT syndrome had been made, lamotrigine was commenced, and the dose was increased to $150 \mathrm{mg}$ twice a day with only a mild reduction in pain. There was a history of hypertension, stroke and seizure disorder secondary to cerebrovascular disease. Seizures were well controlled on phenytoin $300 \mathrm{mg}$ daily. There was no history of migraine or other headache disorder. Other medications included irbesartan, digoxin, clopidogrel, baclofen $25 \mathrm{mg}$ twice a day, and high doses of paracetamol and tramadol.

MRI brain demonstrated an aberrant loop of the right superior cerebellar artery impinging on the trigeminal nerve. Old lacunar infarcts were noted in both cerebral hemispheres and the left pons. MR angiography showed basilar artery stenosis. Lamotrigine was titrated to $200 \mathrm{mg}$ twice a day and phenytoin tapered. Two months later, she was readmitted with intolerable SUNCT pain. Gabapentin (1800 mg per day) and prednisolone (25 mg per day) had no effect. Subcutaneous infusion of $2 \%$ lignocaine in normal saline at $6 \mathrm{ml}$ per hour resulted in symptomatic improvement: she was able to eat without triggering an attack of SUNCT. Following discharge, she was only pain-free for 3 days; increasing frequency and severity of attacks prompted a further admission 1 month later. There was again a good response to lignocaine infusion. Pain recurred on her way home from hospital necessitating readmission and further lignocaine. Clopidogrel was temporarily ceased, and microvascular decompression was performed. Postoperatively, there was imme- diate relief of SUNCT, and no further attacks have occurred after 32 months without prophylactic medication.

\section{DISCUSSION}

We have demonstrated that microvascular decompression using a modified Jannetta procedure is both safe and effective in up to two-thirds of cases of otherwise medically intractable SUNCT/ SUNA when there is evidence of an aberrant artery abutting the symptomatic trigeminal nerve. Complications were minimal and generally short-lived, with only one subject suffering prolonged hearing loss. In this series, cases with SUNCT (71\%) were more responsive to this therapy than those with SUNA (50\%). However, the small numbers make it impossible to draw any firm conclusions in this regard, but this may hint at some differences in the underlying pathophysiology. These results suggest that, in some cases at least, SUNCT and SUNA are due to an external or extra-axial trigger and that these external compressive lesions represent potential therapeutic targets in severe, medically resistant cases.

The pathophysiology of SUNCT, like other trigeminal autonomic cephalalgias, is presumed to involve the trigeminal autonomic reflex. ${ }^{23}$ Impulses from the superior salivatory nucleus travel in the greater superficial petrosal nerve to terminate in the lacrimal system, nasal mucosa and blood vessels liberating vasoactive intestinal peptide. The superior salivatory nucleus is stimulated from impulses from the nucleus caudalis. This system is influenced or activated by an area in the posterior hypothalamus, the so-called internal trigger. Ipsilateral hypothalamic activation has been reported in SUNCT, ${ }^{24}$ but others have demonstrated bilateral hypothalamic activation. ${ }^{25}$ Interestingly, this latter case also had evidence of an ipsilateral aberrant loop of the superior cerebellar artery abutting the trigeminal nerve and has been painfree for 2 years following microvascular decompression. ${ }^{25}$

The surgical management of SUNCT is controversial, and caution has been advised..$^{21-26}$ There have been four case reports of successful microvascular decompression with follow-up periods ranging from 3 months to 2 years. ${ }^{15} 192025$ In three of these reports, a preoperative MRI clearly showed an aberrant vascular compressive loop that was confirmed at surgery. One case cited by Matharu failed to respond to microvascular decompression, but it is unclear whether that case had an identified vascular compression of the trigeminal nerve or not. ${ }^{26}$ Glycerol rhizotomy (one case) and $\gamma$ knife surgery (two cases) have been reported with unsuccessful results. ${ }^{21}$ Radio-frequency thermocoagulation has similarly been unhelpful. ${ }^{26}$ Retro-Gasserian 
glycerol appears to have been initially successful in three cases but had to be repeated in two cases. ${ }^{18}$ Percutaneous compression of the Gasserian ganglion has also been reported as effective. ${ }^{27}$ However, other reports would suggest that trigeminal nerve ablation should be avoided. ${ }^{21}$

In microvascular decompression, the trigeminal nerve is preserved and the source of irritation removed. We used a biologically inert silicone sleeve as a modification of the Janetta procedure to provide protection to the nerve from the irritation of the pulsating abutting artery. This provides long-term separation of the nerve from the artery.

The immediate and dramatic response in the majority of our series suggests a peripheral cause for the symptomatology in a proportion of SUNCT/SUNA cases. The continuing presence of autonomic features, albeit mild in two cases, however, suggests that the central pathways of the trigeminal autonomic reflex can remain active, responding to a light somatosensory trigeminal stimulation. This indicates that an inherently overactive trigeminal autonomic reflex may be a significant underlying mechanism in some patients with SUNCT/SUNA.

Three cases did not improve, despite evidence of successful vascular decompression at surgery. In one of these cases, there had been previous trigeminal nerve damage from herpes zoster infection in addition to arterial compression, and thus there may have been dual pathology. It is interesting to note that the previous marked autonomic features resolved following microvascular decompression, but pain was still as intense as it was prior to surgery. The two remaining treatment failures could be due to either failure to adequately decompress the nerve or other pathophysiological mechanisms predominating.

It could be argued that the surgery only induced or was coincidental to a natural remission in the course of the disease. The period of follow-up was quite significant (mean 22.2 months). Periods of remission are well recognised in SUNCT/SUNA and can last up to 7 years. In seven of our cases, the disease course was chronic, and it has been our experience ${ }^{2}$ and others ${ }^{4}$ that chronic cases do not spontaneously remit or become episodic. Consequently, we believe it is unlikely that coincidental remission is the explanation for these results. The power of the placebo effect should never be dismissed in such uncontrolled studies, particularly those involving a significant procedure as in the present series. The one case of deafness postprocedure, which is a recognised complication of microvascular decompression, was not felt to be related to the use of the silicone sleeve. The apparent effectiveness of microvascular decompression in two-thirds of cases as reported in this study does raise the issue of potential overlap in pathophysiology and clinical phenotype in trigeminal neuralgia and SUNCT/SUNA. However, the authors believe that the prominence of autonomic features and the absence of a refractory period are clinically robust and useful distinguishing features. There may be overlap in the underlying pathophysiology with a variable mixture of peripheral and central mechanisms being prominent in individual cases with both groups of disorders.

In this small proof-of-principle study, we have demonstrated that patients with intractable SUNCT/SUNA and evidence of ipsilateral trigeminal nerve compression due to an aberrant artery can benefit from microvascular decompression using a modified Jannetta procedure. These results can only be viewed as provisional, and further validation through an appropriate randomised trial is required. We would recommend that in resistant cases of SUNCT/SUNA, the trigeminal nerve should be carefully interrogated for any evidence of vascular compression, as this could be the underlying cause.
In the absence of an adequately powered randomised, placebocontrolled trial, we conclude that in medically intractable SUNCT and SUNA syndrome with a demonstrable aberrant arterial loop impinging on the appropriate trigeminal nerve, microvascular decompression is worth considering after exclusion of other causes. The risk of potential complications needs to be weighed up carefully.

Competing interests None.

Patient consent Obtained.

Ethics approval Ethics approval was provided by the Gold Coast Hospital HREC.

Provenance and peer review Not commissioned; externally peer reviewed.

\section{REFERENCES}

1. Headache Classification Subcommittee of the International Headache Society. The international classification of headache disorders: 2nd edition. Cephalalgia 2004;24(Suppl 1):9-160.

2. Williams MH, Broadley SA. SUNCT and SUNA: clinical features and medical treatment. J Clin Neurosci 2008:5:526-34.

3. Goadsby PJ, Cittadini E, Burns B, et al. Trigeminal autonomic cephalalgias: diagnostic and therapeutic developments. Curr Opin Neurol 2008; 21:323-30.

4. Cohen AS, Matharu MS, Goadsby PJ. Trigeminal autonomic cephalalgias: current and future treatments. Headache 2007:47:969-80.

5. Fromm GH. Trigeminal neuralgia and related disorders. Neurol Clin 1989;7:305-19.

6. Pareja JA, Baron M, Gili P, et al. Objective assessment of autonomic signs during triggered first division trigeminal neuralgia. Cephalalgia 2002;22:251-5.

7. Bouhassira D, Attal N, Esteve M, et al. 'SUNCT' syndrome. A case of transformation from trigeminal neuralgia? Cephalalgia 1994;14:168-70.

8. Sesso RM. SUNCT syndrome or trigeminal neuralgia with lacrimation and conjunctival injection? Cephalalgia 2001;21:151-3.

9. Sjaastad 0, Pareja JA, Zukerman E, et al. Trigeminal neuralgia. Clinical manifestations of first division involvement. Headache 1997;37:346-57.

10. Graff-Radford SB. SUNCT syndrome responsive to gabapentin (Neurontin). Cephalalgia 2000;20:515-17.

11. Narbone MC, Gangemi S, Abbate M. A case of SUNCT syndrome responsive to verapamil. Cephalalgia 2005;25:476-8.

12. Porta-Etessam J, Benito-Leon J, Martinez-Salio A, et al. Gabapentin in the treatment of SUNCT syndrome. Headache 2002;42:523-4.

13. Dandy WE. Concerning the cause of trigeminal neuralgia. Am J Surg 1934:24:447-55.

14. Leone M, Rigamonti A, Usai S, et al. Two new SUNCT cases responsive to lamotrigine. Cephalalgia 2000;20:845-7.

15. Gardella L, Viruega A, Rojas $H$, et al. A case of a patient with SUNCT syndrome treated with Jannetta procedure. Cephalalgia 2001;21:996-9.

16. Koseoglu E, Karaman Y, Kucuk S, et al. SUNCT syndrome associated with compression of trigeminal nerve. Cephalalgia 2005;25:473-5.

17. Cohen AS, Matharu MS, Goadsby PJ. Short-lasting unilateral neuralgiform headache attacks with conjunctival injection and tearing (SUNCT) or cranial autonomic features (SUNA)-a prospective clinical study of SUNCT and SUNA. Brain 2006;129:2746-60.

18. Hannerz J, Linderoth B. Neurosurgical treatment of short-lasting, unilateral, neuralgiform hemicrania with conjunctival injection and tearing. $\mathrm{Br} \mathrm{J}$ Neurosurg 2002:16:55-8

19. Lagares A, Gomez PA, Perez-Nunez A, et al. Short-lasting unilateral neuralgiform headache with conjunctival injection and tearing syndrome treated with microvascular decompression of the trigeminal nerve: case report. Neurosurgery 2005;56:E413; discussion E413.

20. Lenaerts M, Diederich N, Phuoe D. A patient with SUNCT cured by the Jannetta procedure. Cephalalgia 1997;17:460.

21. Black DF, Dodick DW. Two cases of medically and surgically intractable SUNCT: a reason for caution and an argument for a central mechanism. Cephalalgia 2002;22:201-4.

22. Rovit RL, Murali R, Jannetta PJ. Trigeminal neuralgia. Baltimore, MD: Williams \& Wilkins, 1990.

23. Goadsby PJ, Lipton RB. A review of paroxysmal hemicranias, SUNCT syndrome and other short-lasting headaches with autonomic feature, including new cases. Brain 1997; 120(Pt 1):193-209.

24. May A, Bahra A, Buchel C, et al. Functional magnetic resonance imaging in spontaneous attacks of SUNCT: short-lasting neuralgiform headache with conjunctival injection and tearing. Ann Neurol 1999:46:791-4.

25. Sprenger T, Valet M, Platzer S, et al. SUNCT: bilateral hypothalamic activation during headache attacks and resolving of symptoms after trigeminal decompression. Pain 2005;113:422-6.

26. Matharu MS, Cohen AS, Goadsby PJ. SUNCT syndrome responsive to intravenous lidocaine. Cephalalgia 2004;24:985-92.

27. Morales-Asin F, Espada F, Lopez-Obarrio LA, et al. A SUNCT case with response to surgical treatment. Cephalalgia 2000;20:67-8. 